\title{
Instructions and decision times of size-constancy responses
}

\author{
DALE W. KAESS \\ University of Aberdeen, Aberdeen AB $2 U B$, Scotland
}

\begin{abstract}
The current study investigated the effect of instructions and stimulus discriminability on size-constancy responses and decision times in a task allowing separate evaluation of the effects of instructions and of dimensional choices by the subjects in the size-constancy task. Objective, perspective, or distance matches were influenced both by the instructions given the subject and by the relative discriminability of the stimuli in each dimension, so that subjects did not always successfully follow their instructions. Decision times were found to be fastest with perspective rather than objective or apparent instructions, but times taken to produce objective and perspective matches did not differ. Apparently, both objective and perspective judgments are available to the perceiver before a response is made, but instructions affect postperceptual response times.
\end{abstract}

There is now a substantial literature concerning the effects of instructions on size-constancy performance (cf. Carlson, 1977, for a review). Generally, instructions to judge the objective size of a stimulus produce larger size estimates than do instructions to judge its perspective size. It is apparent that subjects attempt to judge that aspect of the stimulus (objective or perspective size) described in their instructions. A third sort of instruction, called "phenomenal," does not specify any particular aspect of the stimulus. Instead, size estimates are to be based upon an immediate impression of how the stimulus looks to the subject. Since he obtained larger size estimates with these phenomenal instructions than with either objective or perspective instructions, Landauer (1966) concluded that phenomenal instructions produced an entirely different perceptual set than those produced by the other two. In particular, since they were largest, phenomenal size judgments must not be the result of confusion between objective and perspective responses. Thus, there are apparently at least three unique sorts of instructions for the size-constancy task.

Recent research has shown that the different instructions may produce different response latencies as well as different size judgments. Epstein and Broota (1975) found that a longer time was required for judgments under objective- than under phenomenalsize instructions. It has also been shown that decision times in size constancy become greater with increased distance of the stimulus (Broota, 1975; Broota \& Epstein, 1973; Epstein \& Broota, 1975; Gulick \& Stake, 1957). However, decision times for size estimation varied with distance only with objective in-

The author wishes to thank Martha Teghtsoonian and the anonymous reviewers, who provided helpful editorial comments, and C. R. Kaess, who tested the subjects and provided advice and support throughout the project. structions, and decision times for judgments of distance per se did not vary as distance varied. Therefore, Epstein and Broota (1975) concluded that the observed differences in decision time probably reflected postperceptual response effects produced by the implied veridicality demands of the instructions. Objective instructions specifically describe the possible influence of distance upon perceived size, and may imply that caution is required in judgments. Phenomenal instructions, in contrast, request size estimates based on an immediate impression, which might not involve consideration of distance by the perceiver. This view is consistent with those theories which assume that instructions do not affect the perceptual processing of information but, rather, bias the responses given once perceptual processing is complete (e.g., Carlson, 1977).

Another factor that might affect decision times in size constancy is the degree of uncertainty with different instructions and stimuli. Kaess $(1971,1973$, 1978) has shown that subjects in the shape-constancy task are influenced by the relative discriminability of the objective, perspective, or angle variation among stimuli. In the most direct test (Kaess, 1978), a design similar to that of tests of dimensional dominance (e.g., Odom \& Guzman, 1972; Smiley, 1972a, 1972b) was used. Subjects were instructed to choose either the wider objective width or the wider perspective width of two simultaneously presented rectangles. The relative discriminability of differences between widths on the two dimensions was also varied. Instructions to respond to a dimension were successful only in the conditions in which the relative differences between objective and perspective widths were approximately equal. But when discriminability of differences on the two dimensions was not equal, responses were nearly always to the dimension that was 
more similar to that trained, regardless of instructions specifying a dimension. Thus, subjects do not necessarily attend only to that dimension described in their instructions, but may instead respond on the basis of the relative discriminability or salience of the information available to them on each trial. Consequently, comparisons among instruction groups' performance may be only a crude measure of differences among reactions to aspects of the stimuli.

The present study has two major objectives. The first aim is to demonstrate that the type of judgmentobjective, perspective, or phenomenal-made by the subject depends not only on the instructions, but also on the relative discriminability of objective and perspective size differences. A series of trials of the type often used to test dimensional preferences by children are used. On each trial, a standard stimulus is presented together with a pair of comparison forms. One comparison form is identical to the standard form in objective size, perspective size, or distance, but differs from it on the other two dimensions. The second comparison form is identical on a second dimension, and each dimension is used equally often with each of the three instructional sets. Thus, it is possible to determine to which dimension of stimulus variation-objective, perspective, or distancethe subject responds most often during the experiment. The relative discriminability of differences between values of the standard and comparison stimuli has been systematically varied to show that the subject will often identify the stimulus by the most discriminable values, regardless of instructions to attend to any particular dimension.

The second aim is to show that decision times in the size-constancy task are a function of relative discriminability of the stimuli. Studies that appear to demonstrate that response times are longer by groups given objective-size instructions than by those given phenomenal-size instructions (Epstein \& Broota, 1975 ) or that decision times become longer with increased distance of the stimuli (Broota, 1975; Broota \& Epstein, 1973; Epstein \& Broota, 1975) may have confounded instructions and stimulus discriminability.

\section{METHOD}

\author{
Subjects \\ Thirty-six paid adult volunteers served as subjects. All had \\ normal vision.
}

\section{Stimuli and Apparatus}

Stimuli were $26 \times 20 \mathrm{~cm}$ black-and-white prints and $35-\mathrm{mm}$ black-and-white slides, showing three black triangles on a table covered with a checkerboard pattern. Behind the stimuli was a vertical backdrop of the same checkerboard pattern. The center figure was an equilateral triangle, $5 \mathrm{~cm}$ on a side, placed at $50 \mathrm{~cm}$ distance (Exemplar 1) or at $100 \mathrm{~cm}$ distance (Exemplar 2). On each side of this standard form were two other triangles that differed according to each of three test sets. The objective sizes, distances, and visual angles subtended by these comparison stimuli for the Exemplar 1 standard are presented in Table 1. Each test set was designed so that one comparison form possessed an identical value to the standard on one dimension but differed by a ratio of $1: 1.2$ or $1: 1.5$ on another dimension. The respective dimensions were reversed for the second comparison form. Thus, in test set OP. Triangle 1 was identical to the standard in objective size $(5 \mathrm{~cm})$ and Triangle 2 was identical to it in visual angle (5.7 deg), while both differed from the standard form in the other dimension and in distance. A choice of Triangle 1 was denoted objective, and a choice of Triangle 2, perspective. In test set OD, Triangle 1 (the objective choice) was of identical objective size as the standard $(5 \mathrm{~cm})$ but differed from it in distance (and thus visual angle), while Triangle 2 (the distance choice) was at the same distance as the standard form $(50 \mathrm{~cm})$ but differed from it in objective size (and thus in visual angle). Distance or objective size differed by the ratio of $1: 1.2$ or of $1: 1.5$, as in the other tests. Finally, comparison stimuli of test set $P D$ varied perspective size (visual angle) and distance in the same manner. A choice of Triangle 1 was denoted perspective, and a choice of Triangle 2 , distance. There were four photographs for each test set with each exemplar standard, comprising an orthogonal set of two levels of discriminability from the standard on each of the two dimensions tested in the set.

\section{Procedure}

The $35-\mathrm{mm}$ slides were back-projected onto a $100 \times 60 \mathrm{~cm}$ screen placed about $1.5 \mathrm{~m}$ away from the subject. The prints were handed to the subject over a $20-\mathrm{cm}$ wall placed on the table at which the subject was seated, and the subject held the prints while viewing them. A timer started as the print appeared in the subject's view. The subject chose either the right or left triangle by pressing appropriately marked buttons on a panel in front of him or her. Decision times were recorded automatically when one of these buttons was pressed. Each subject judged both slides and prints, in orders balanced over subjects within each group. All subjects judged stimuli of all three sets, each set including stimuli at two levels

Table 1

Sizes (Length of Side in Centimeters), Distances (in Centimeters), and Visual Angles (in Degrees) of the Triangle in Each Test

\begin{tabular}{|c|c|c|c|c|c|c|c|c|c|c|c|c|}
\hline \multirow{3}{*}{$\begin{array}{c}\text { Stimulus } \\
\text { Photo- } \\
\text { graph }\end{array}$} & \multicolumn{4}{|c|}{ OP Test } & \multicolumn{4}{|c|}{ OD Test } & \multicolumn{4}{|c|}{ PD Test } \\
\hline & \multicolumn{2}{|c|}{ Triangle 1} & \multicolumn{2}{|c|}{ Triangle 2} & \multicolumn{2}{|c|}{ Triangle 1} & \multicolumn{2}{|c|}{ Triangle 2} & \multicolumn{2}{|c|}{ Triangle 1} & \multicolumn{2}{|c|}{ Triangle 2} \\
\hline & Size & VA & Size & VA & Size & Distance & Size & Distance & VA & Distance & VA & Distance \\
\hline 1 & 5.0 & 3.8 & 6.0 & 5.7 & 5.0 & 60 & 6.0 & 50 & 5.7 & 60 & 6.9 & 50 \\
\hline 2 & 5.0 & 3.8 & 7.5 & 5.7 & 5.0 & 60 & 7.5 & 50 & 5.7 & 60 & 8.6 & 50 \\
\hline 3 & 5.0 & 4.8 & 6.0 & 5.7 & 5.0 & 75 & 6.0 & 50 & 5.7 & 75 & 6.9 & 50 \\
\hline 4 & 5.0 & 4.8 & 7.5 & 5.7 & 5.0 & 75 & 7.5 & So & 5.7 & 75 & 8.6 & 50 \\
\hline
\end{tabular}

Note $-V A=$ visual angle. Standard triangle for each pair was an equilateral triangle $5 \mathrm{~cm}$ on a side at $50-\mathrm{cm}$ distance (VA = 5.7 deg).

In addition, the same objective sizes were presented at twice these distances, to produce a similar set of objective, perspective, and distance relationships but with smaller visual angles. 
of discriminability of each of two dimensions and at two distances of the standard form, with each comparison form to the left and right of the standard, using both slides and prints. Each condition was repeated four times. Thus, each subject received a total of 384 trials.

There were three instruction groups of 12 subjects each. The objective instruction group was asked to choose the form on the right or left which was most similar to the center one in its physical size, while the perspective instruction group was asked to choose the more similar perspective (visual angle) size. These two groups were given a thorough description of the differences between perspective and physical sizes and were asked to attend only to the dimension of their instructions. The third, phenomenal instruction, group was asked to choose the form which "looked like" the center form, with no description of physical and perspective sizes or distances given.

Slides and prints, rather than three-dimensional objects, were used to avoid differences in reaction times produced by accommodation and convergence (see Massaro, 1973). Size constancy is possible with such pictorial arrays (Leibowitz, Bussey, \& McGuire, 1957; Winters \& Baldwin, 1971). However, it might be expected that more perspective image matches would be obtained with slides than with three-dimensional displays. Furthermore, since the prints were not presented at the orthoposition (at which distance, etc., would be most accurately represented), prints might be expected to produce more perspective matches than would slides. Inspection of the data, to be reported later, reveals, however, that there were $t_{1} O$ significant differences between the number of choices of the perspective dimension with slide and print stimuli.

\section{RESULTS}

The data analyses are described in two sections. First, the number of objective, perspective, and distance choices by each instruction group for slides and prints are considered with respect to the relative discriminability of the dimensions. The purpose of this analysis is to show that the extent to which subjects attend to specific dimensions in the size constancy experiment depends upon the relative discriminability of the dimensions of variation of the stimuli. Second, the decision times for objective, perspective, and distance choices are analyzed to show the differences in decision times for correct and incorrect responses, as a function of the instructions given to the subjects.

The number of choices of the left or right triangle in the three tests was tabulated to obtain the total number of objective, perspective, and distance matches in all trials for each subject. Since the task was a forced-choice one with 384 trials per subject, and since each of the tabulated numbers results from two tests, each involving forced choice trials with one of the other dimensions as the alternative choice, the numbers of objective, perspective, and distance choices are not mutually independent. Consequently, the data are analyzed by three separate (partially dependent) analyses of variance. The numbers of objective, perspective, and distance responses were subjected to separate, 3 (instruction group) by 2 (slide-print stimulus presentation) by 2 (relevant dimension discriminability) by 2 (irrelevant dimension discriminability) analyses, with 12 subjects per cell. Table 2 contains the $F$ ratios resulting from these analyses,
Table 2

Summary of F Ratios from Analyses of Variance of Objective, Perspective, and Distance Choices

\begin{tabular}{|c|c|c|c|c|}
\hline \multirow[b]{2}{*}{$\begin{array}{l}\text { Source of } \\
\text { Variance }\end{array}$} & \multirow[b]{2}{*}{ df } & \multicolumn{3}{|c|}{ Response } \\
\hline & & $\begin{array}{c}\text { Objec- } \\
\text { tive }\end{array}$ & $\begin{array}{l}\text { Perspec- } \\
\text { tive }\end{array}$ & $\begin{array}{c}\text { Dis- } \\
\text { tance }\end{array}$ \\
\hline Instructions (I) & 2,33 & $4.467^{*}$ & $11.360^{*}$ & $3.960 *$ \\
\hline Stimuli (S) & 3 & $7.016^{*}$ & 1.0 & $4.457^{*}$ \\
\hline I $\times S$ & 2,33 & .643 & $2.606^{*}$ & $4.023^{*}$ \\
\hline Relevant Dimension $(\mathrm{R})$ & 1,33 & $88.124^{*}$ & $74.057^{*}$ & $4.196^{*}$ \\
\hline I $x S \times R$ & 2,33 & .460 & $4.401^{*}$ & .962 \\
\hline Irrelevant Dimension (IR) & 1,33 & $21.287^{*}$ & $14.672^{*}$ & $92.645^{*}$ \\
\hline I x IR & 2,33 & $8.403^{*}$ & 1.650 & 1.371 \\
\hline$R \times I R$ & 1,33 & .277 & $17.965^{*}$ & $8.982^{*}$ \\
\hline I $\times$ R x IR & 2,33 & .685 & $5.404^{*}$ & $4.399^{*}$ \\
\hline$I \times S \times R \times I R$ & 2,33 & $6.989^{*}$ & .220 & $4.799^{*}$ \\
\hline
\end{tabular}

${ }^{*} p<.05$.

with those sources of variance not resulting in significantly large $(p>.05)$ ratios excepted. As can be seen in Table 2 , instructions produced a significantly large effect on each of the three dimensional responses. Objective instructions produced the most objective and distance responses, and perspective instructions produced the most perspective responses. The discriminability of both the relevant and the irrelevant dimensions also had a significantly large effect on each of the dimensional choices. In each case, there were significantly more choices of a dimension when that dimension was more discriminable, and significantly fewer choices of a dimension when the irrelevant dimensions were more discriminable. The discriminability of the relevant and irrelevant dimensions interaction effect, as well as the triple interaction involving instructions, was significantly large with the perspective and distance dimensional choices, but not with the objective dimensional choices. The relevant by irrelevant dimension discriminability interaction with each type of instruction is shown in Figure 1. Finally, more objective dimension choices and fewer distance dimension choices were made with slides than with prints, but this effect of stimulus presentation was not large with perspective dimension choices. The remaining significantly large effects do not result from any simple pattern of means and are probably not important to the conclusions of the present study.

In summary, the results of these analyses of number of objective, perspective, and distance dimension choices are: (1) Subjects were influenced by the instructions given them; most objective and most perspective dimension choices were made by those groups given objective and perspective instructions, respectively, with phenomenal instructions producing an intermediate number of choices on each dimension. (2) Increased discriminability of the relevant dimension (the dimension described in the instructions) resulted in an increased number of choices, and increased discriminability of the irrelevant dimension (the dimension 

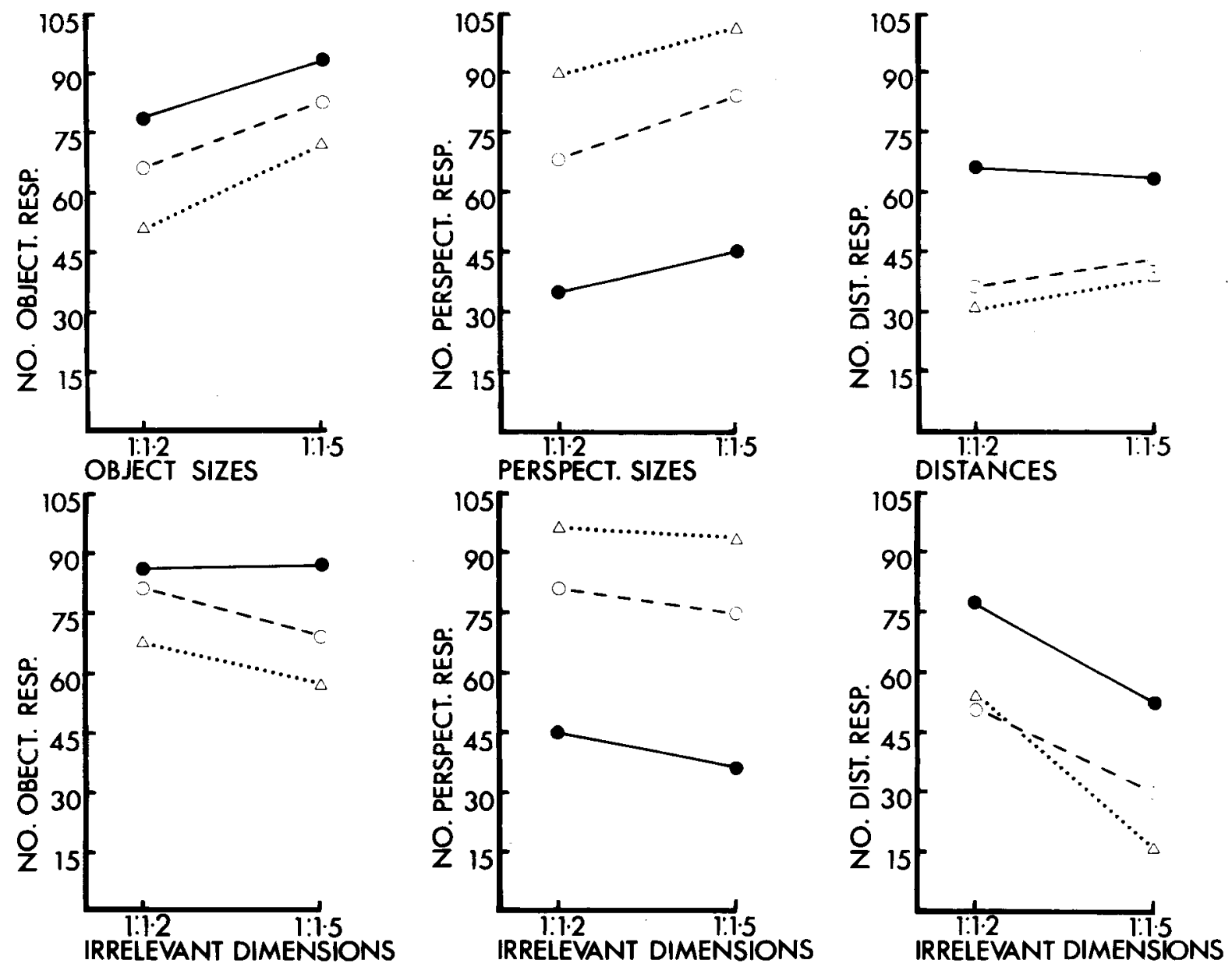

Figure 1. Mean number of objective, perspective, and distance responses (columns 1, 2, and 3, respectively) for objective (solid line), phenomenal (dashed line), and perspective (dotted line) instructions. In the top row, responses are shown as a function of the discriminability of the relevant dimension, and in the bottom row, of the irrelevant dimension.

associated with the relevant dimension but not instructed, on each two-choice task) decreased the number of choices of each instructed dimension. (3) The interaction of relevant vs. irrelevant dimension discriminability and the triple interaction of these factors with instructions were large with the perspective and distance choices, but not with the objective choices. The conclusion is that the subjects apparently understood the instructions given them, since most objective or perspective choices were made with objective or perspective instructions, respectively. But the extent to which these instructions were successfully followed depends upon the relative discriminability of the stimuli along the various dimensions. Figure 1 illustrates this effect of dimensional discriminability on responses following each sort of instruction.

The average number of choices and the average decision times for each type of dimensions choice are presented in Table 3 . It can be seen by comparison of column means in Table 3 that, over all instruction groups, about an equal number of objective and perspective choices were made. Thus, there was no general tendency to "prefer" one of these dimensions, but both objective and perspective choices were made more frequently than were distance choices. Apparently, subjects interpreted all three sorts of instruction to call for a size match (either objective or perspective size), rather than for a match of the distances.

The second analysis involved comparisons of the decision times taken to make objective, perspective, or distance choices, with each type of instruction. Since there was little effect of mode of stimulus presentation (slides/prints) revealed in the first set of analyses, the mode of stimulus presentation was not included in this second set of analyses. All subjects made some choices of each of the objective, perspective, and distance dimensions, so that there were no null cells in this analysis of decision times.

The average decision times for each type of dimension choice were subjected to a 2 (instructions) by 3 
Table 3

Average Decision Time (DT) and Proportion (P) of Matches (in Percentages)

for Three Types of Response Under Three Types of Instruction

\begin{tabular}{lccccccc} 
& \multicolumn{2}{c}{ Objective } & \multicolumn{2}{c}{$\begin{array}{c}\text { Response } \\
\text { Phenomenal }\end{array}$} & \multicolumn{2}{c}{ Perspective } \\
\cline { 2 - 7 } Instruction & DT & P & DT & P & DT & P & Mean \\
\hline Objective & 258.56 & 45 & 284.30 & 21 & 299.89 & 34 & 280.92 \\
Phenomenal & 223.56 & 39 & 233.52 & 40 & 254.43 & 21 & 237.26 \\
Perspective & 202.28 & 32 & 188.77 & 50 & 217.20 & 18 & 202.75 \\
Mean & 228.23 & 39 & 235.53 & 37 & 257.17 & 24 & \\
\hline
\end{tabular}

(dimension choice) analysis of variance. Both the simple effects of instruction group $[\mathrm{F}(2,33)=3.849$, $\mathrm{p}<.05]$ and of dimensional choice $[\mathrm{F}(2,66)=14.306$, $\mathrm{p}<.001]$ were significantly large. As Epstein and Broota (1975) found, objective instructions required longer decision times than either phenomenal or perspective instructions. However, only the decision times to make distance dimensional choices differed from those to the other dimensions. Decision times to make objective dimension choices, while slightly faster, were not significantly different from decision times to make perspective dimension choices [t(35) $<1.0]$. Thus, while the perspective instructions produced overall faster responding, the actual choices of the perspective dimension were not made faster than were choices of the objective dimension. The Instruction by Dimensional Choice interaction effect was not significantly large $[F(4,66)=2.130, p>.05]$.

\section{DISCUSSION}

There are several important implications for the understanding of size-constancy performance derived from the present findings. First, subjects do not consistently respond as instructed. This conclusion is analogous to that of Kaess (1978) concerning shapeconstancy performance. While the instructions given the subjects do have an important effect on their performance (possibly the most important effect; cf. Carlson, 1977), other factors such as the relative discriminability of the dimension of variation also strongly influence the size- and shape-constancy performance.

Given the usual objective, phenomenal, or perspective size-constancy instructions, subjects apparently attempt to judge either objective or perspective size rather than distance. There were fewest choices of the distance dimension, and the choices made required longer decision times. Many choices of distance may have been errors in judging the objective or perspective sizes, and the longer decision times for distance choices may reflect the uncertainty associated with judgments on these trials. No instructions to match on the basis of distance were given in the present experiment. Epstein and Broota (1975) found that subjects instructed to judge distances responded faster than those instructed to judge objective size; how- ever, it is not known with what frequency they followed those instructions. Such frequency information would be important for understanding the related phenomena of distance constancy (cf. Ono \& Comerford, 1977) or speed constancy (cf. Epstein, 1978).

Phenomenal instructions, which do not specify either objective or perspective size choices, produce an approximately equal number of each. This finding is interesting when considered together with Landauer's (1966) results. Landauer obtained larger size judgments with phenomenal instructions. Thus, apparently the phenomenal size is greater than the objective size of the form, and subjects of the present experiments might have perceived the objective size alternative to be more nearly the appropriate match to the phenomenal size than was the smaller, perspective size alternative. However, subjects of the phenomenal instruction group responded either to objective size or to perspective size, doing so about equally often. The mean judged sizes, were they measured in the present experiment, could only have been in agreement with Landauer's findings if subjects made gross overestimations of objective and perspective sizes under phenomenal instructions. Since size judgments were not obtained, the present data do not allow evaluation of this possibility, however.

Finally, the major finding of the present study is that Epstein and Broota's (1975) conclusion that instructions affect postprocessing time in size constancy requires clarification. The present results seem to confirm Epstein and Broota's finding of longer decision times with objective than with phenomenal instructions (and extend those findings to perspective instructions). But the present results also reveal that the effect of instructions on decision times is a matter of response accuracy: Actual objective and perspective size matches are made with equal decision times. The apparently longer decision times required by objective instructions result from a greater number of errors produced by the subject given those instructions. Perhaps the same effect accounts for Epstein and Broota's finding that objective instructions produce longer decision times than do phenomenal instructions. If this is the case, their conclusion that instructions affect postprocessing time in size constancy may require modification. 


\section{REFERENCES}

Broota, K. D. Chronometric study in making absolute size and distance judgments. Indian Journal of Psychology, 1975, 50, 58-66.

Broota, K.D., \& Epstein, W. The time it takes to make veridical size and distance judgments. Perception \& Psychophysics, $1973,14,358-364$.

CARLSON, V. R. Instructions and perceptual constancy judgments. In W. Epstein (Ed.), Stability and constancy in visual perception: Mechanisms and processes. New York: Wiley-Interscience, 1977.

EPSTEIN, W. The process of 'taking-into-account' in visual perception. Perception, 1973, 2, 267-285.

EPSTE IN, W. Two factors in the perception of velocity at a distance. Perception \& Psychophysics, 1978, 24, 105-114.

Epstein, W., \& Broota, K. D. Attitude of judgment and reaction time in estimation of size at a distance. Perception \&. Psychophysics, 1975, 18, 201-204.

Gulick, W. L., \& Stake, R. E. The effect of time on sizeconstancy. American Journal of Psychology, 1957, 70, 276-279.

KaEss, D. W. Analytic and intuitive perceivers in the form constancy task. Psychonomic Science, 1971, 23, 179-180.

KAESS, D. W. The invariance hypothesis and the intuitive perceiver: A developmental study. Perception, 1973, 2, 3-9.

KAESS, D. W. Importance of relative width differences and instructions on shape constancy performance. Perception, 1978, 7, 179-186.

Landauer, A. A. A note on the effect of instructions on size judgments with the tunnel illusion. Australian Journal of Psychology, 1966, 18, 144-147.
Leibowitz, H., Bussey, T., \& McGuire, P. Shape and size constancy in photographic reproductions. Journal of the Optical Society of America, 1957, 47, 658-661.

Massaro, D. W. The perception of rotated shapes: A process analysis of shape constancy. Perception \& Psychophysics, 1973, $13,413-422$.

Odom, R. D., \& Guzman, R. D. Development of hierarchies of dimensional salience. Developmental Psychology, 1972, 6, 271-287.

Ono, H., \& Comerford, J. Stereoscopic depth constancy. In W. Epstein (Ed.), Stability and constancy in visual perception: Mechanisms and processes. New York: Wiley-Interscience, 1977.

SmiLey, S. S. Instability of dimensional preference following changes in relative cue similarity. Journal of Experimental Child Psychology, 1972, 13, 394-403. (a)

Smiley, S. S. Optional shift behavior as a function of dimensional preference and relative cue similarity. Journal of Experimental Child Psychology, 1972, 14, 313-322. (b)

Winters, J. J., \& Baldwin, D. Development of two- and threedimensional size constancy under restricted cue conditions. Journal of Experimental Psychology, 1971, 88, 113-118.

WoHLwill, J. F. The development of "overconstancy" in space perception. In L. P. Lipsitt \& C. C. Spiker (Eds.), Advances in child development and behavior (Vol. 1). New York: Academic Press, 1963.

(Received for publication December 11, 1978; revision accepted February 13, 1980.) 